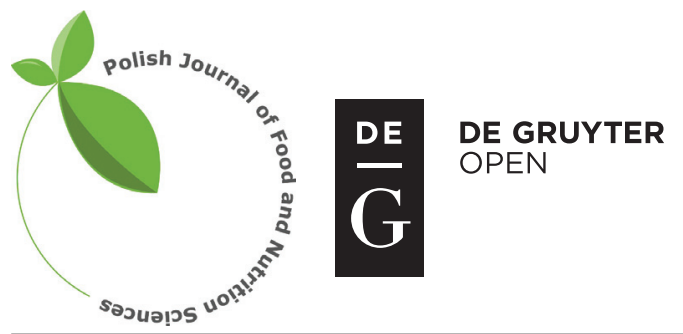

Pol. J. Food Nutr. Sci., 2018, Vol. 68, No. 4, pp. 299-308

DOI: $10.2478 /$ pjfns-2018-0002 http://journal.pan.olsztyn.pl

Original research article

Section: Food Quality and Functionality

\title{
Effects of Anthocyanin-Rich Popping Maize Flour on the Phenolic Profile and the Antioxidant Capacity of Mix-Bread and Its Physical and Sensory Properties
}

\author{
Marijana Simić1, Slađana Žilićc ${ }^{*}$, Olivera Šimuruna ${ }^{3}$, Bojana Filipčev ${ }^{3}$, Dubravka Škrobot ${ }^{3}$, Jelena Vančetović \\ ${ }^{1}$ Department of Food Technology and Biochemistry, Maize Research Institute, Slobodana Bajica 1, Belgrade-Zemun, Serbia \\ ${ }^{2}$ Plant Breeding Department, Maize Research Institute Slobodana Bajica 1, Belgrade-Zemun, Serbia \\ ${ }^{3}$ Institute of Food Technology, Bulevar cara Lazara 1, Novi Sad, Serbia
}

Key words: anthocyanin-rich popping maize flour, bread, phenolic profile, anthocyanins, antioxidant capacity, physical and sensory properties

\begin{abstract}
In this study, the effect of anthocyanin-rich popping maize flour on phenolic profile, antioxidant capacity and color of bread were investigated Texture and sensory properties of maize mix-breads were evaluated also. Replacing $30 \%$ of wheat flour with blue- and dark-red-seeded maize flours increased the content of total phenolic compounds, ferulic and $p$-coumaric acids of mix-breads compared to the control bread. In addition, anthocyanins from maize flours improved functional profile of breads. Despite thermal degradation, the content of anthocyanins in crumb of blue and dark-red maize mix-breads was high and amounted to $142.3 \mathrm{mg} \mathrm{CGE} / \mathrm{kg}$ and $84.4 \mathrm{mg} \mathrm{CGE} / \mathrm{kg}$, respectively. In his acylated and non-acylated forms, cyanidin 3-glucoside was the most prevalent anthocyanins in maize mix-breads. However, given to the synergistic and antagonistic interaction that arises from the coexistence of numerous antioxidant compounds in food, anthocyanin-rich maize flour had no effect on the increase in the bread antioxidant capacity.

Anthocyanin-rich maize flour changed the bread crumb color to a red range. Although maize mix-breads had worse textural properties compared to the wheat bread, their sensory quality was improved.
\end{abstract}

\section{INTRODUCTION}

There is a growing recognition that the bioactive compounds present in food have beneficial effects on human health through their antioxidant actions [Dey et al., 2016]. Due to the putative role in the prevention of various diseases associated with oxidative stress, phenolic compounds have become especially interesting for researchers, food manufacturers and consumers. A current estimated dietary intake of total phenolic compounds is $>1 \mathrm{~g}$ /day [Scalbert \& Williamson, 2000]. This intake is about 10 times higher than that of vitamin C, more than 100 times higher than that of vitamin $\mathrm{E}$ and about 500 times higher than that of $\beta$-carotene [Hooper \& Cassidy, 2006]. Fruits and beverages are the main dietary sources of phenolic compounds. Cereals also contribute to the phenolics intake, particularly of insoluble bound phenolic forms. However, due to the removal of phenolic-rich bran and the aleurone layer during whole grains milling, cereal products, such as wheat bread, are scarce in these phytochemicals. Given that bread is a major staple wheat-based food product widely accepted among consumers in the world and that it is predominantly rich in carbohydrates, it is the food suitable for fortification with bioactive compounds, essential micro- and macronutrients, as well as an appropriate vehicle for their delivery. There have been many

\footnotetext{
* Corresponding Author: Tel: +381 113756 704; Fax: +381 113754 994;

E-mail: szilic@mrizp.rs (Dr. Slađana Žilić)
}

studies concerning the enrichment of wheat bread with different small grain cereals, cereal by-products and pseudo-cereals, as well as spices, fruits and herbs in the order to produce bread with improved antioxidant properties [Yousif et al., 2012; El-Megeid et al., 2009]. However, studies of blue and dark-red maize flour used for this purpose are rare.

Anthocyanins are the main antioxidants in colored maize grains. Studies have shown that the main anthocyanins present in colored maize were identified as cyanidin-3-glucoside, pelargonidin-3-glucoside, and peonidin-3-glucoside, along with their corresponding malonyl derivatives [Žilić et al., 2012]. Recent results of Mora-Rochín et al. [2016] have shown that the acyl type anthocyanins, such as cyanidin-3-(6-succinyl glucoside) and cyanidin-3-(6-disuccinyl glucoside) were the most abundant compounds in blue colored maize, accounting for $52.1 \%$ and $15.6 \%$ the total anthocyanins, respectively. It should be noted that monomeric anthocyanins possess limited stability against hydration, $\mathrm{pH}$ changes, temperature and light exposure [Dangles et al., 1993] while recent studies have shown that the anthocyanins with acylating substituents were more stable during processing and storage [Bąkowska-Barczak, 2005]. A partial substitution of white wheat flour with wholegrain anthocyanin-rich maize flour can greatly improve functional properties, as well as the nutritional value of bread. Nevertheless, it poses considerable technological difficulties because maize proteins lack the ability to form the necessary gluten network for holding the gas produced during dough fermentation. 
The objective of this study was to investigate the effect of blue- and dark-red-seeded popping maize flour on the phenolic profile and the antioxidant capacity of mix-breads, as well as their physical characteristics and sensory properties.

\section{MATERIAL AND METHODS}

\section{Plant materials}

The wheat (Triticum aestivum L.) genotype ZP 87/I and two popping maize (Zea mays spp. everta) genotypes recently developed at the Maize Research Institute, Zemun Polje (MRIZP), in the vicinity of Belgrade, Serbia, were used. One blue- and one dark-red-seeded maize genotype were used. All genotypes were grown in the field at the Maize Research Institute, Belgrade (4452' N, 20¹9’ E, $82 \mathrm{~m}$ a.s.1.), Serbia, in the 2016 growing season. Standard cropping practices were applied to provide adequate nutrition and to keep the disease- and weed-free plots.

\section{Flour samples preparation}

Wheat flour $(<180 \mu \mathrm{m})$ was produced in a milling company (Žitoprodukt d.o.o. Belgrade-Batajnica, Serbia) in the experimental mill (Laboratory mill; Bühler MLU-202, Uzwil, Switzerland). The characteristics of the obtained flour correspond to those of flour with 0.45 to $0.55 \%$ of ash. In contrast to the wheat flour that was debranned, the wholegrain maize flour was used. Grains of blue and dark-red-seeded popping maize were ground on a Perten 120 lab mill (Perten Instruments, Hägersten, Sweden) to powder $(<500 \mathrm{~mm})$. Flour samples were prepared immediately prior to bread-making.

\section{Bread-making procedure}

Three types of bread samples were prepared in this study based on different wheat/maize flour ratios and maize genotype used: wheat flour bread (100:0 w/w), wheat and blue popping maize bread (70:30), and wheat and dark-red popping maize bread $(70: 30 \mathrm{w} / \mathrm{w})$. A basic bread formula, based on flour weight, was used as follows: flour (300 g), yeast (7.5 g), salt $(6.0 \mathrm{~g})$, vegetable fat $(3.0 \mathrm{~g})$, and water according to farinograph absorption. The ingredients were mixed for $5 \mathrm{~min}$ in a laboratory Diosna mixer and the dough was left for ripening (40 min) at $85 \%$ relative humidity and $30 \pm 1^{\circ} \mathrm{C}$. Then, the dough was divided into $115 \pm 1 \mathrm{~g}$ portions, manually rounded, rolled and put into tin pans $(9.5 \times 7.5 \mathrm{~cm} \times$ $5.5 \mathrm{~cm}$ ). The final fermentation lasted $43 \mathrm{~min}$ for wheat bread and $35 \mathrm{~min}$ for bread with added blue and red maize flour. The breads were baked at $230^{\circ} \mathrm{C}$ for $20 \mathrm{~min}$ in a deck type oven (Miwe condo, D-97450, Arnstein, Germany). All baking experiments were performed in four repetitions. Baked breads were chilled and stored at $24^{\circ} \mathrm{C}$ and relative humidity of $34 \%$ for $24 \mathrm{~h}$ and then bread quality was evaluated. Bread crumb samples and manually separated upper crusts were lyophilized and used for analyses of phenolic compounds.

\section{Analytical procedures}

\section{Extraction of phenolic compounds from flour and bread}

For the detection of the total phenolics, total flavonoids and phenolic acids extracts were prepared from $0.5 \mathrm{~g}$ of flour or bread. All flour and lyophilized bread samples were stored in dark at $-18^{\circ} \mathrm{C}$ before analysis to protect bioactive components from degradation. The extraction of phenolic compounds was performed within 10 days. After alkaline hydrolysis for $4 \mathrm{~h}$ at room temperature using $10 \mathrm{~mL}$ of $4 \mathrm{M} \mathrm{NaOH}$, extraction was done with ethyl acetate and diethyl ether (1:1, $\mathrm{v} / \mathrm{v}$ ) four times. Five $\mathrm{mL}$ of combined extracts were evaporated under $\mathrm{N}_{2}$ stream at $30^{\circ} \mathrm{C}$ to dryness and final residues were redissolved in methanol. The extracts were kept at $-70^{\circ} \mathrm{C}$ until analyses. All extractions were performed in duplicate per each replications of the baking experiment.

\section{Analysis of total phenolic content (TPC)}

The total phenolic content was determined according to the Folin-Ciocalteu procedure [Singleton et al., 1999]. Briefly, the extract $(270 \mu \mathrm{L})$ was transferred into a test tube and the volume adjusted to $500 \mathrm{~mL}$ with distilled water and oxidized with the addition of $250 \mu \mathrm{L}$ Folin-Ciocalteu reagent. After $5 \mathrm{~min}$, the mixture was neutralized with $1 \mathrm{~mL}$ of $20 \%$ aqueous $\mathrm{Na}_{2} \mathrm{CO}_{3}$ solution. After $40 \mathrm{~min}$, the absorbance was measured at $725 \mathrm{~nm}$. The total phenolic content was expressed as $\mathrm{mg}$ of gallic acid equivalent (GAE) per kg of dry matter (d.m.).

\section{Analysis of total flavonoid content (TF)}

The total flavonoid content was determined according to Eberhardt et al. [2000]. The solution of $5 \% \mathrm{NaNO}_{2}(50 \mu \mathrm{L})$ was mixed with $200 \mu \mathrm{L}$ of extract. After $6 \mathrm{~min}, 500 \mu \mathrm{L}$ of a $10 \%$ $\mathrm{AlCl}_{3}$ solution was added, and after $7 \mathrm{~min}, 250 \mu \mathrm{L}$ of $1 \mathrm{M}$ $\mathrm{NaOH}$ was added. Absorbance was measured at $510 \mathrm{~nm}$. The total flavonoid content was expressed as mg of catechin equivalent (CE) per $\mathrm{kg}$ of d.m.

\section{Analysis of total anthocyanin content (TAC)}

Anthocyanins were extracted from $80 \mathrm{mg}$ of maize flour and $250 \mathrm{mg}$ of bread samples mixing with $5 \mathrm{~mL}$ of methanol acidified with $1 \mathrm{M} \mathrm{HCl}(85: 15$, v/v). After shaking, the absorbance was measured at 535 and $700 \mathrm{~nm}$ [Abdel-Aal \& Hucl, 1999]. The content was expressed as mg of cyanidin 3-glucoside equivalent (CGE) per kg of d.m.

\section{Analysis of individual phenolic acids}

Chromatographic analyses were performed on the Thermo Scientific Ultimate 3000 HPLC with a photodiode array detector (Thermo Scientific, Waltham, Massachusetts, USA). Phenolic acids were separated on the Thermo Scientific Hypersil GOLD aQ C18 column $(150 \mathrm{~mm} \times 4.6 \mathrm{~mm}$, i.d., $3 \mu \mathrm{m})$ using a linear gradient elution program with a mobile phase containing solvent A (formic acid $/ \mathrm{H}_{2} \mathrm{O}, 1: 99, \mathrm{v} / \mathrm{v}$ ) and solvent $\mathrm{B}$ (methanol) at a flow rate of $0.8 \mathrm{~mL} / \mathrm{min}$. The solvent gradient was programmed as described by Žilić et al. [2012]. The chromatograms were recorded at $280 \mathrm{~nm}$ by monitoring spectra within the wavelength range of 190-400 $\mathrm{nm}$. Identified phenolic acid peaks were confirmed and quantified using the Thermo Scientific Dionex Chromeleon 7.2. chromatographic software.

\section{Analysis of individual anthocyanins}

Depending on the sample, 80 to $500 \mathrm{mg}$ were mixed with $1.5 \mathrm{~mL}$ of methanol acidified with $1 \mathrm{M} \mathrm{HCl}(85: 15$, v/v) in three subsequent extractions. After centrifugation (Dina- 
myca Pty Ltd, Clayton Victoria, Australia) at 10,000 rpm for 3 min the supernatants were collected. In order to confirm the presence of acylated forms of anthocyanin, the alkaline hydrolysis of the anthocyanin extracts was carried out with $10 \% \mathrm{KOH}$. The hydrolysates were kept in darkness for $10 \mathrm{~min}$ and then $6 \mathrm{M} \mathrm{HCl}$ was added to the solution until stabile red color appeared. Both, anthocyanin extracts and their hydrolysates were filtrated through nylon syringe filter of $0.45 \mu \mathrm{m}$ and used for the chromatographic analysis. The HPLC analysis was carried out with Thermo Scientific UltiMate 3000 HPLC system. Chromatograms were obtained at $530 \mathrm{~nm}$ after injection of $10 \mu \mathrm{L}$ of sample. Separation was performed on a Thermo Scientific Hypersil GOLD aQ C18 column $(150 \mathrm{~mm} \times 4.6 \mathrm{~mm}$, i.d., $3 \mu \mathrm{m})$ using a linear gradient elution program with a mobile phase containing solvent A (formic acid/ $\mathrm{H}_{2} \mathrm{O}, 1: 99, \mathrm{v} / \mathrm{v}$ ), and solvent $\mathrm{B}$ (formic acid/acetonitrile, $1: 99, \mathrm{v} / \mathrm{v}$ ) at a flow rate of $0.7 \mathrm{~mL} / \mathrm{min}$ and the column oven temperature of $30^{\circ} \mathrm{C}$. The following gradient was established: linear gradient elution from $10 \% \mathrm{~B}$ to $30 \% \mathrm{~B}, 0-30$ min; linear gradient elution from $30 \% \mathrm{~B}$ to $100 \% \mathrm{~B}, 30-35 \mathrm{~min}$; isocratic elution of $100 \%$ B, 35-38 min; linear gradient elution from $100 \% \mathrm{~B}$ to $10 \% \mathrm{~B}, 38-40 \mathrm{~min}$; isocratic elution of $10 \% \mathrm{~B}$, 40-45 min. Pure anthocyanin compounds such as delfinidin 3-glucoside, cyanidin 3,5-diglucoside, cyanidin 3-glucoside, petunidin 3-glucoside, pelargonidin 3-glucoside, and malvidin 3-glucoside produced by Sigma-Aldrich (Taufkirchen, Germany) were used as standards. Identified anthocyanin peaks were confirmed and quantified using the Thermo Scientific Dionex Chromeleon 7.2. chromatographic software.

\section{Analysis of the total antioxidant capacity $(A C)$}

The total antioxidant capacity was measured according to the direct or QUENCHER method described by Serpen et al. [2008] using the ABTS (2,2-azino-bis 3-ethil-benothiazoline6 -sulfonic acid) reagent. The total antioxidant capacity was expressed as mmol Trolox equivalents (Eq) per kg of d.m.

\section{Measurement of color}

Color properties of flour and bread samples were measured with a chroma meter (CR-400/410, Konica, Minolta, Japan), which was calibrated against white calibration standard (CM -A70). The surface color of samples were given as average $L^{*}$ (lightness), $a^{*}$ (redness), $b^{*}$ (yellowness) values of four measurements per samples.

\section{Analysis of physical properties of bread}

Bread loaf volumes $(\mathrm{mL})$ and specific volumes $(\mathrm{mL} / \mathrm{g})$ were determined by VolScan profiler (Stable Microsystem, Surrey, UK). The hardness, chewiness, cohesiveness, resilience and springiness of bread crumbs were instrumentally recorded on a texture analyzer TA.XT plus (Stable Micro Systems, England, UK) equipped with a $36 \mathrm{~mm}$ cylindrical probe according to AACC method 7410A modified as described by Filipčev et al. [2010].

\section{Sensory analysis of bread}

The sensory properties included the crumb elasticity and appearance of crumb pores (structure and uniformity, Dallman pores), as well as bread shape, smell, taste, aroma and chewiness. After $24 \mathrm{~h}$ baking, the breads were sliced and evaluated on the 5-point intensity scale by a 6 -member panel whose members had been trained in the sensory evaluation. Sensory properties of bread samples were evaluated by the 5-point category scale with end-points labelled from 1 to 5 as described by Filipčev et al. [2010].

\section{Statistical analysis}

The data were reported as mean \pm standard deviation of at least four independent repetitions. The results were statistically analyzed using the Statistica software version 5.0 (StatSoft Co., Tulsa, OK, USA). Significance of differences between samples was analyzed by the Tukey's test (HSD). Differences at $\mathrm{p}<0.05$ were considered significant.

\section{RESULTS AND DISCUSSION}

\section{Phenolic compounds content, the antioxidant capacity and the color of wheat and maize flour}

The content of total phenolic compounds, flavonoids, total anthocyanins, as well as phenolic acids and individual anthocyanins in both wheat and maize flour is given in Table 1. On average, in wheat flour the content of total phenolic compounds and flavonoids was about 8-fold and 3.3-fold lower than that in blue and dark-red popping maize flour, respectively (Table 1). Since the only white color is primary genetic characteristic of hexaploid wheat, it is quite understandable that anthocyanins were not found in wheat flour (Table 1). In complete contrast to wheat flour, the wholegrain maize flour was rich in anthocyanins. Although dark-red popping maize flour had a considerably higher content of total phenolic compounds than those from blue popping maize, higher total anthocyanins content was measured in blue popping maize flour ( $824 \mathrm{mg} \mathrm{CGE/kg} \mathrm{d.m.).} \mathrm{The} \mathrm{observed}$ differences in total anthocyanins content could be attributed primarily to the location of anthocyanins in the maize grain, and depending on that to their varying availability. The chromatographic profile for the anthocyanins indicated the presence of six anthocyanins in maize flours (Figure 1a). However, comparing retention times and UV-visible spectral data with known available standards, the presence of cyanidin 3-glucoside and pelargonidin 3-glucoside were confirmed. Peaks 3-6 did not match any of the anthocyanin standards. The most common quantified anthocyanin in blue and darkred maize flour was cyanidin 3-glucoside (137.5 and $95.8 \mathrm{mg}$ / $\mathrm{kg}$, respectively) (Table 1), which is in agreement with results reported by Žilić et al. [2012]. The content of second major defined anthocyanin pelargonidin 3-glucoside was on average a 6.5 -fold lower than that of cyanidin 3-glucoside glucoside in the both maize flour samples. Previous investigations [dePascual-Teresa et al., 2002; Pedreschi \& Cisneros-Zevallos, 2007] showed that the retention times for the acylated and aglycone forms of anthocyanins was longer than the retention time for the anthocyanin glycosides found. The comparison of our chromatographic profiles and retention times (Figure 1a) with the same of aforementioned authors, shows that they were quite similar. Further, the presence of acylated forms was confirmed by the basic hydrolysis of the methanolic extract (data not shown). According to Pedreschi \& 
TABLE 1. The content of phenolic compounds and antioxidant capacity of cereal flours, wheat bread and maize mix-breads.

\begin{tabular}{|c|c|c|c|c|c|c|c|c|}
\hline \multirow[b]{2}{*}{ Samples } & \multirow{2}{*}{$\begin{array}{c}\text { TPC } \\
(\mathrm{mg} \mathrm{GAE} / \mathrm{kg})\end{array}$} & \multirow{2}{*}{$\begin{array}{c}\mathrm{TF} \\
(\mathrm{mg} \mathrm{CE} / \mathrm{kg})\end{array}$} & \multirow{2}{*}{$\begin{array}{c}\text { TAC } \\
(\mathrm{mg} \mathrm{CGE} / \\
\mathrm{kg})\end{array}$} & \multicolumn{2}{|c|}{ Phenolic acids } & \multicolumn{2}{|c|}{ Anthocyanins } & \multirow{2}{*}{$\begin{array}{c}\mathrm{AC} \\
(\mathrm{mmol} \text { Trolox } \\
\mathrm{Eq} / \mathrm{kg})\end{array}$} \\
\hline & & & & $\begin{array}{c}\text { Ferulic acid } \\
(\mathrm{mg} / \mathrm{kg})\end{array}$ & $\begin{array}{l}p \text {-Coumaric } \\
\text { acid }(\mathrm{mg} / \mathrm{kg})\end{array}$ & $\begin{array}{c}\mathrm{C} 3 \mathrm{G} \\
(\mathrm{mg} / \mathrm{kg})\end{array}$ & $\begin{array}{c}\mathrm{Pg} 3 \mathrm{G} \\
(\mathrm{mg} / \mathrm{kg})\end{array}$ & \\
\hline \multicolumn{9}{|c|}{ Flour* } \\
\hline Wheat & $340 \pm 17^{c}$ & $91.9 \pm 1.2^{\mathrm{C}}$ & n.d. & $48.6 \pm 0.8^{\mathrm{C}}$ & n.d. & n.d. & n.d. & $19.9 \pm 0.3^{\mathrm{C}}$ \\
\hline Blue popping maize & $2564 \pm 59^{\text {в }}$ & $344 \pm 18^{\mathrm{A}}$ & $824 \pm 1^{\mathrm{A}}$ & $4096 \pm 30^{\mathrm{A}}$ & $333 \pm 11^{\mathrm{B}}$ & $137.5 \pm 7.1^{\mathrm{A}}$ & $20.6 \pm 1.7^{\mathrm{A}}$ & $21.7 \pm 0.2^{\mathrm{B}}$ \\
\hline Red popping maize & $2882 \pm 41^{\mathrm{A}}$ & $284 \pm 19^{\mathrm{B}}$ & $574 \pm 2^{\mathrm{B}}$ & $3417 \pm 29^{\text {в }}$ & $389 \pm 21^{\mathrm{A}}$ & $95.8 \pm 4.2^{\mathrm{B}}$ & $14.4 \pm 1.4^{\mathrm{B}}$ & $36.8 \pm 0.3^{\mathrm{A}}$ \\
\hline \multicolumn{9}{|c|}{ Bread $^{* *}$} \\
\hline W (crumb) & $327 \pm 16^{c}$ & $64.2 \pm 0.1^{\mathrm{a}}$ & n.d. & $43.1 \pm 3.0^{c}$ & n.d. & n.d. & n.d. & $18.7 \pm 0.8^{\mathrm{ab}}$ \\
\hline W (crust) & $469 \pm 27^{c}$ & $22.6 \pm 0.4^{\mathrm{f}}$ & n.d. & $41.1 \pm 2.1^{\mathrm{c}}$ & n.d. & n.d. & n.d. & $21.4 \pm 2.1^{\mathrm{a}}$ \\
\hline $\begin{array}{l}\text { 70\%W+30\%B.P.M. } \\
\text { (crumb) }\end{array}$ & $1291 \pm 12^{\mathrm{b}}$ & $51.4 \pm 1.0^{\mathrm{b}}$ & $142.3 \pm 0.5^{\mathrm{a}}$ & $1051 \pm 46^{\mathrm{a}}$ & $82.3 \pm 1.6^{\mathrm{b}}$ & $42.7 \pm 0.1^{\mathrm{a}}$ & $6.89 \pm 0.07^{\mathrm{a}}$ & $17.9 \pm 1.0^{\mathrm{b}}$ \\
\hline $\begin{array}{l}70 \% \mathrm{~W}+30 \% \text { B.P.M. } \\
\text { (crust) }\end{array}$ & $1639 \pm 13^{\mathrm{a}}$ & $38.3 \pm 1.2^{\mathrm{d}}$ & $81.9 \pm 1.6^{\mathrm{b}}$ & $1027 \pm 57^{\mathrm{a}}$ & $81.4 \pm 0.4^{b}$ & $32.6 \pm 0.2^{\mathrm{b}}$ & $2.99 \pm 0.48^{\mathrm{b}}$ & $21.3 \pm 0.5^{\mathrm{a}}$ \\
\hline $\begin{array}{l}\text { 70\%W+30\% R.P.M. } \\
\text { (crumb) }\end{array}$ & $1350 \pm 8^{b}$ & $45.9 \pm 0.4^{c}$ & $84.4 \pm 0.6^{\mathrm{b}}$ & $993 \pm 59^{a}$ & $106.9 \pm 7.4^{\mathrm{a}}$ & $29.8 \pm 0.2^{c}$ & $3.61 \pm 0.06^{\mathrm{b}}$ & $17.9 \pm 1.0^{\mathrm{b}}$ \\
\hline $\begin{array}{l}70 \% \mathrm{~W}+30 \% \text { R.P.M. } \\
\text { (crust) }\end{array}$ & $1323 \pm 15^{\mathrm{b}}$ & $35.3 \pm 0.7^{\mathrm{e}}$ & $54.1 \pm 0.6^{c}$ & $900 \pm 26^{\mathrm{b}}$ & $99.6 \pm 0.8^{\mathrm{a}}$ & $22.8 \pm 0.1^{\mathrm{d}}$ & $1.70 \pm 0.03^{c}$ & $21.3 \pm 0.9^{\mathrm{a}}$ \\
\hline
\end{tabular}

*content is expressed per $\mathrm{kg}$ of dry matter of flour; ${ }^{* *}$ content is expressed per kg of dry matter of bread. TPC - total phenolic compounds, TF - total flavonoids, TAC - total anthocyanins, C3G - cyanidin 3-glucoside, Pg3G - pelargonidin 3-glucoside, AC -antioxidant capacity, W - wheat, B.P.M. - blue popping maize, R.P.M. - dark-red popping maize, n.d.- not detected. The content of TPC, TF and phenolic acids was determined after base hydrolysis of crude samples. Means followed by the same letter (lower case) within the same column are not significantly different $(p>0.05)$. Means followed by the same letter (upper case) within the same column are not significantly different ( $p>0.05$ ). Letters correspond to ranking of groups after Tukey's test.

Cisneros-Zevallos [2007], the six anthocyanin glucosides and the respective acylated forms were detected in Andean purple maize. In our study, a relative area of the peak with the retention time at 14.36 min (peak 4) was about 3 -fold higher than that of cyanidin 3-glucoside in both pigmented maize flours. These compounds, which corresponded to acylated cyanidin 3-glucoside, represented the major derivate of anthocyanin in blue and dark-red maize flours accounting for about $48-52 \%$ of the total peaks area of anthocyanins. It was followed by the non-acylated cyanidin 3-glucoside that represented about $17 \%$ of the total peaks area of anthocyanins. These results are consistent with those of Mora-Rochín et al. [2016]. Compared to acylated anthocyanins, the color contribution of monomeric non-acylated forms is lesser [Stintzing et al., 2002]. The results of these authors show that the color impact of cyanidin 3-glucoside, as the major compound of blackberry extract, was only about $50 \%$. According to our results, anthocyanins of blue and dark-red popping maize at $\mathrm{pH}$ of about 5.4 provided bright blue and pale red color of flour (Figure 2), respectively.

Although ferulic and $p$-coumaric acids are the primary and most abundant phenolic acids in cereals [Irmak et al., 2008; Montilla et al., 2011; Žilić et al., 2012], p-coumaric acid was not detected in wheat flour (Table 1). The content of ferulic acid in wheat flour amounted to $48.6 \mathrm{mg} / \mathrm{kg}$ that was 84-fold and 70-fold lower than that found in blue and darkred popping maize flour, respectively. It is noticeable that the total content of identified phenolic acids in maize flour samples was high in comparison with their content of total phenolic compounds measured by the Folin-Ciocalteu as- say. Use of gallic acid for the expression of the total phenolic compounds content is one of the possible reasons for discrepancies that have arisen. In addition to the flavonoids and phenolic acids, it was evident that some other bioactive compounds contributed to the antioxidant capacity of wheat flour. The antioxidant capacity of phenolic-poor wheat flour was $19.9 \mathrm{mmol}$ Trolox Eq/kg, while the anthocyaninrich blue popping maize flour had the antioxidant capacity of $21.7 \mathrm{mmol}$ Trolox Eq $/ \mathrm{kg}$. The highest antioxidant capacity correlated with the highest content of total phenolic compounds in dark-red popping maize flour (Table 1).

\section{Phenolic compounds content, the antioxidant capacity and the color of bread crumb and crust}

Having in mind thermal energy transfer trough bread, and effects of temperature, as a dominating factor on the baking mechanisms including gelatinization, enzymatic activity, yeast growth and browning reaction, the content of phenolic compounds and the antioxidant capacity was measured in the crumb and crust of breads. In order to evaluate functional advantages of enriched maize mix-bread, all the results were compared with those of wheat bread as a control. As expected, replacing $30 \%$ of wheat flour with wholegrain blue and dark-red maize flour had a positive impact on phenolic compounds content in bread and its color (Table 1, Figure 2). On average, the crumb and crust of both enriched breads had about 4-fold and 3-fold higher contents of total phenolic compounds in comparison to that in the control sample, respectively. The content of ferulic acid was by 22 to 25 -fold higher in maize mix-bread samples than that in the wheat bread 

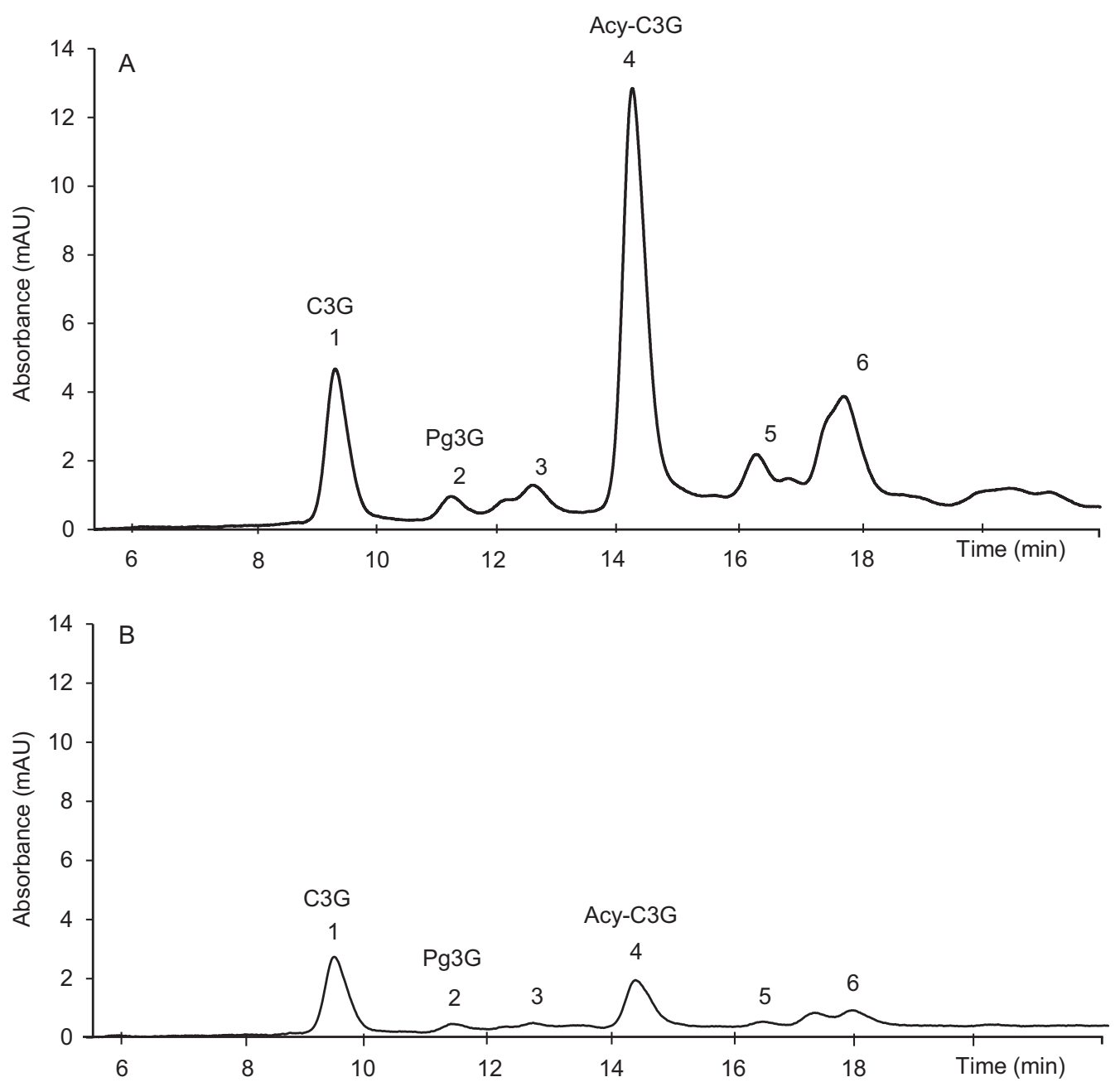

FIGURE 1. HPLC chromatograms of anthocyanins from: A) dark-red maize flour; B) dark-red maize mix-bread crumb. Peaks are noted as follows: 1 - cyanidin 3-glucoside (C3G); 2 - pelargonidin 3-glucoside (Pg3G); 3 - glycosidic form of anthocyanin; 4 - acylated cyanidin 3-glucoside (Acy-C3G); and 5 and 6 - acylated anthocyanins.

samples, while $p$-coumaric acid was not detected in the wheat bread, and its entire content in the enriched bread came from maize (Table 1). Menga et al. [2010] reported that the content of ferulic acid was about 2-fold and 3-fold higher in bread samples obtained by wheat flour enriched with 6 and $10 \%$ of bran than that in control sample, respectively. Similarly, Świeca et al. [2017] have found that the bread enriched with $5 \%$ of green coffee had by 2 -fold higher content of ferulic acid than common wheat bread after in vitro digestion. However, similar to our results, the authors state that the entire content of $p$-coumaric acid in enriched bread originated from coffee.

According to our results, the baking process increased the content of total phenolic compounds in bread samples in comparison to those in composite flours having in mind their percentage share in bread. This is most easily observed in the case of wheat bread. The content of total phenolic compounds in wheat bread crust was by $30 \%$ higher than that in the initial raw material. These increases can be, to some extent, a consequence of bound phenolic acid release during baking. However, it should be mentioned that the Folin-Ciocalteu reagent, used for the analysis of total phenols, may react with any reducing substance. Žilić et al. [2016] stated that the Maillard reaction products which have reductone struc- tures contributed to the increase in total phenolic compounds in cookies measured by the Folin-Ciocalteu assay. Unlike the phenolic acids, flavonoids primarily occur in the conjugated form that makes them less thermally stable. During baking, temperature increases from 150 to $205^{\circ} \mathrm{C}$ in bread crust, while in the bread crumb does not exceed $100^{\circ} \mathrm{C}$. Therefore, a significant reduction in the content of total flavonoids was detected especially in bread crusts in the comparison to composite flours. This reduction in wheat bread crust amounted to $55.6 \%$. Despite the degradation, the content of total anthocyanins in crumb of blue and dark-red maize mix-breads was relatively high and amounted to $142.3 \mathrm{mg}$ CGE/ $\mathrm{kg}$ and $84.4 \mathrm{mg}$ $\mathrm{CGE} / \mathrm{kg}$, respectively, which is a considerable enrichment of wheat bread in terms of its functionality and health effects. The thermal degradation of anthocyanins was more intensive in crusts of mix-bread samples than in their crumbs (Table 1). Cyanidin 3-glucoside was the most prevalent anthocyanin in maize mix-bread samples. However, the percentage contribution of its acylated form to the total peaks area of anthocyanins was significantly reduced in the samples of blue and dark-red maize mix-breads compared to that in the flours amounting to 43 and $37 \%$ in bread crumbs and 30 and $25 \%$ in bread crusts, respectively (Figure 1b). Although the con- 

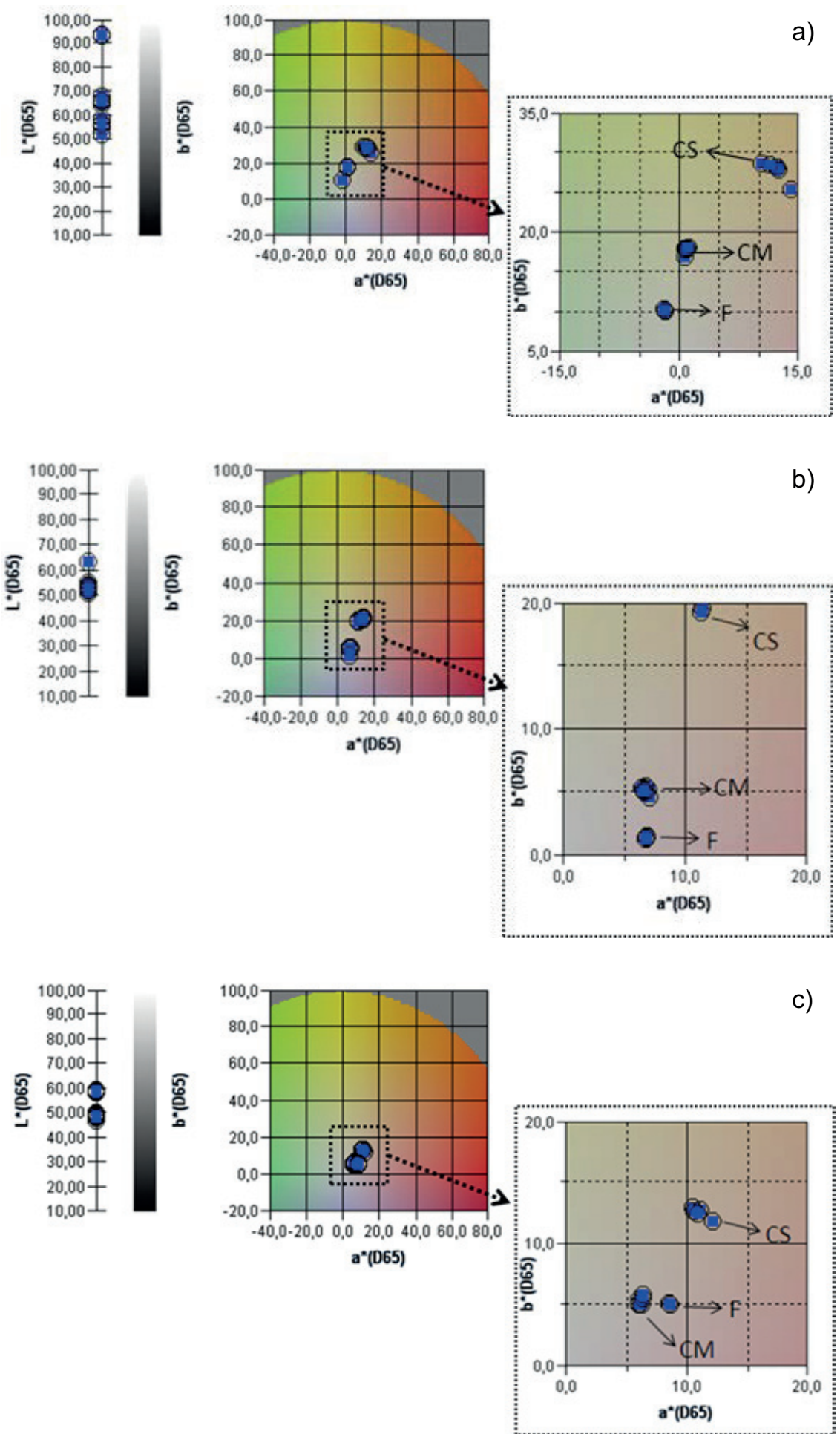

FIGURE 2. CIE $\mathrm{a}^{*}, \mathrm{~b}^{*}$ and $\mathrm{L}^{*}$ chromaticity diagram for: a) wheat flour, wheat bread crust and wheat bread crumb; b) blue maize flour, blue maize mixbread crust and blue maize mix-bread crumb; c) dark-red maize mix-bread crust and dark-red maize mix-bread crumb. F-flour; CS-crust; CM-crumb.

tent of non-acylated glucoside of cyanidin was significantly reduced in maize mix-bread samples, its percentage contribution to the total peaks area of anthocyanins of bread crumbs and crusts was about 2 to 2.5-fold higher in comparison with those in maize flours (Figure 1a and 1b). West \& Mauer [2013] noted that the results for cyanidin 3-glucoside in the presence of an acylated derivative, as occurs in certain purple maize extracts, could be erroneously high. Namely, according to the study of these authors about $68 \%$ of the purified cyanidin 3-(6"-malonoyl) glucoside was lost during storage at $40^{\circ} \mathrm{C}$ and converted primarily to cyanidin 3-glucoside. $\mathrm{Xu}$ [2013] reported that mono-acylated anthocyanins showed a higher resistance against heat than di- and non-acylated. Anthocyanins mono-acylated with $p$-hydroxybenzoic acid were best retained after heating, followed by ferulic acid and caffeic acid. According to our results, the replacement of $30 \%$ of wheat 

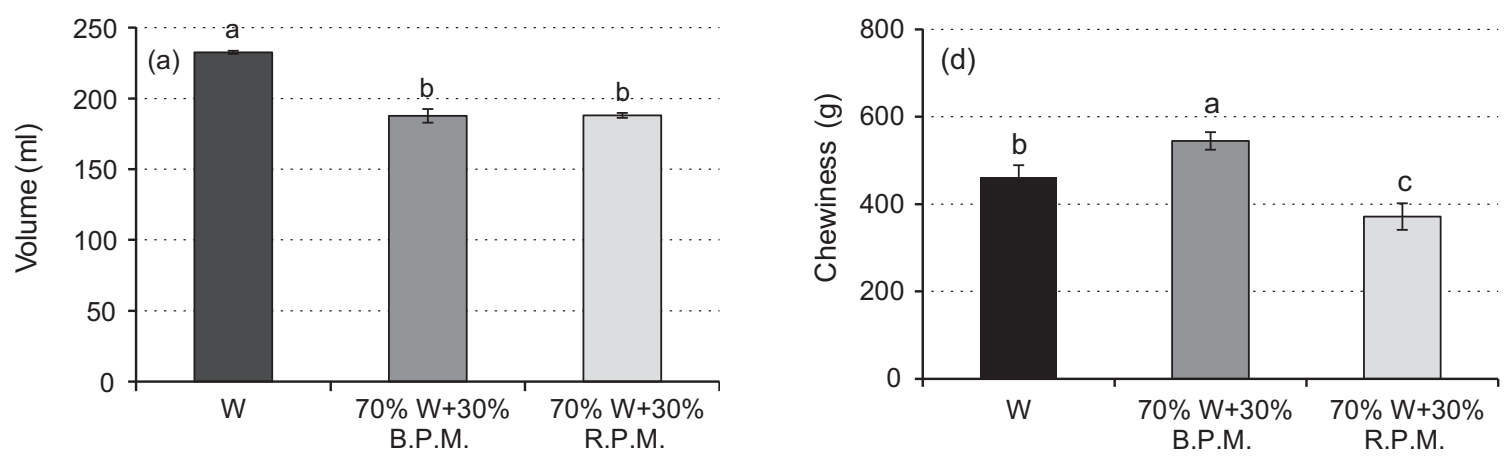

(b)
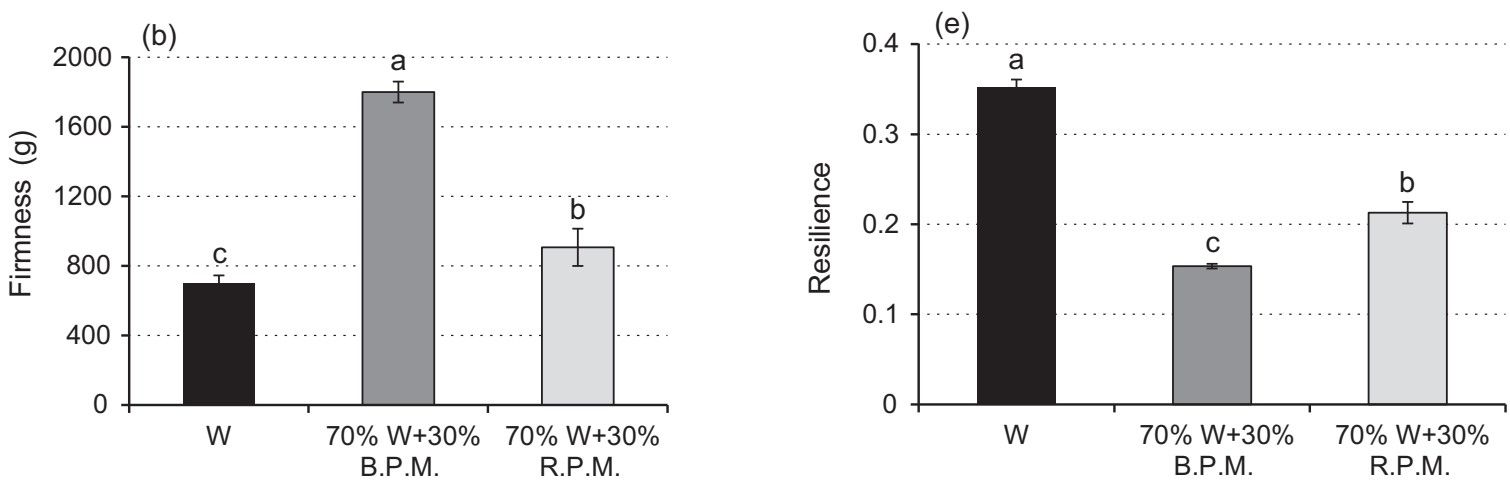

(c)

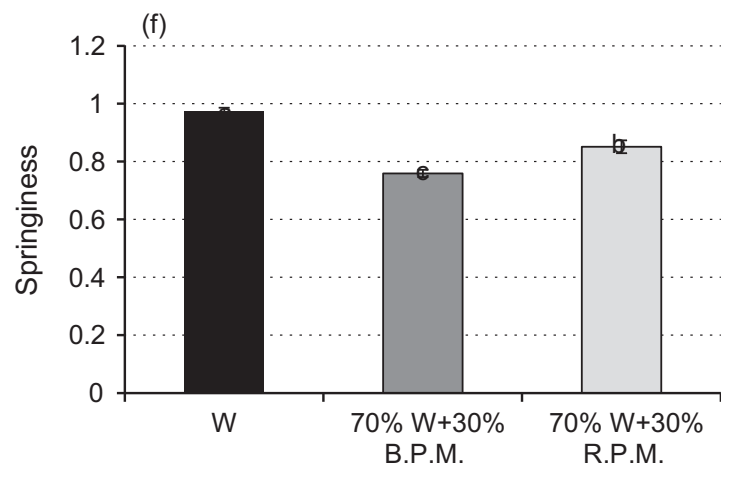

FIGURE 3. Texture properties of wheat bread and maize mix-breads. a) volume; b) firmness; c) cohesiveness; d) chewiness; e) resilience; f) springiness. W- wheat; B.P.M. - blue popping maize; R.P.M. - dark-red popping maize. The vertical bars represent the standard deviation of each data point. Bars with different letters are statistically significantly different, according to Tukey's test $(\mathrm{p}<0.05)$.

flour with anthocyanin-rich popping maize flour had no effect on the mix-bread antioxidant capacity (Table 1). Although dark-red maize had almost two times higher antioxidant capacity compared to wheat flour used, antioxidant capacity of wheat bread and maize mix-bread crumbs, as well as crusts was quite uniform. This could be explained by the synergistic and antagonistic interaction that arises from the coexistence of numerous antioxidant compounds in food. On the other hand, the baking process had a great effect on the antioxidant capacity of bread samples. A higher temperature in bread crusts than that in bread crumbs during baking contributed to the breads surface antioxidant capacity that was by $13 \%$ higher compared to the one in the inner part of breads. This study confirms previous results of Žilić et al. [2016], who showed that the Maillard reaction had a crucial influence on the increment of antioxidant capacity in cookies.

The addition of anthocyanin-rich maize flour changed the bread crumb color to a red range. Crumbs of blue and dark-red maize mix-bread had CIE $a^{*}$ values more than 6-fold higher than wheat bread crumb (Figure 2). In addition, wheat bread crumb had yellow color values (CIE b*) higher by $71.5 \%$ and $70.5 \%$ than crumbs of blue and dark-red bread samples, respectively. The basic difference in the crumbs color of two breads enriched with blue and dark-red maize flour was in CIE L* values. The dark-red maize mix-bread had darker crumb. Generally, bread samples enriched with maize flours had a darker crust than wheat bread. The brown color can be result of thermal degradation of anthocyanins, as well as the formation of brown polymers as the end-product of the Maillard reaction. A high CIE b* value of control wheat bread crust indicated its intense yellow color, which may be due to the intensity of the intermediate stages of the Maillard reaction [Hofmann, 1998], and may partly be due to the thermal oxidation of shortening. According to Kocadağl et al. [2016], $\alpha$-dicarbonyl compound trapping ability of phenolic compounds was attributed to the reduction of some 
a)

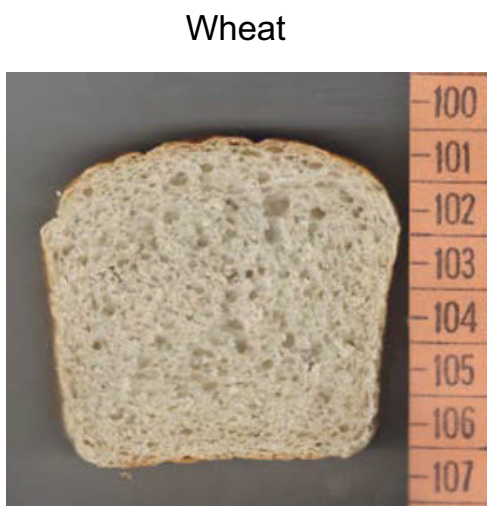

b)

$70 \% \mathrm{~W}+30 \%$ B.P.M.

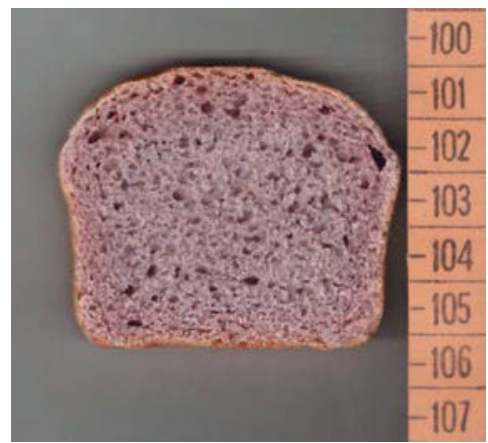

c)

$70 \% \mathrm{~W}+30 \%$ R.P.M.

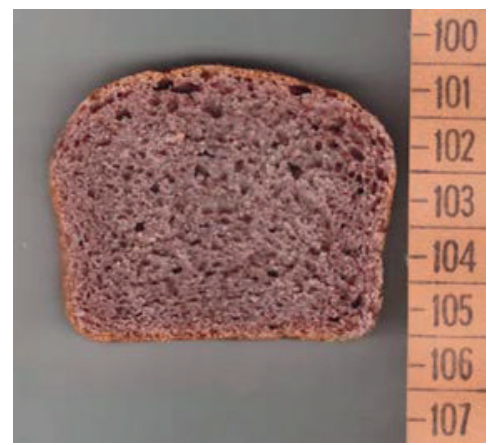

FIGURE 4. Cross-section of breads: a) wheat bread; b) blue maize mix-bread; c) dark-red maize mix-bread. W- wheat; B.P.M. - blue popping maize; R.P.M. - dark-red popping maize.

reactive Maillard reaction intermediates during baking of cookies, that, in our case, could be reflected in significantly lower CIE b* values of maize mix-bread crusts compared with the value of wheat bread crust.

\section{Physical and sensory properties of bread samples}

The results of the texture analysis of wheat and maize mix-breads are presented in Figures 3 and 4. These results indicate that the substitution of wheat flour with maize flour reduced the baking potential in terms of the loaf volume. As a consequence of the addition of gluten-free flour, samples with $30 \%$ of blue and dark-red popping maize flour showed a volume reduction of 20\% compared to wheat bread (Figure 3a). According to Eduardo et al. [2013], bread volume directly influences the firmness of bread. The texture analysis showed a significantly higher firmness of mix-breads when compared to control bread $(\mathrm{p}<0.05)$ with maximum values for blue maize mix-bread (Figure 3b). Firmness of this mix-bread was 2.6 and 2-fold higher than that of wheat bread and dark-red maize mix-bread, respectively. Considering that wheat and maize flour have a quite different water absorption capacity, the addition of maize flour influenced the crumb structure by retaining more moisture, thus increasing the hardness values [Siddiq et al., 2009]. High crumb firmness caused the crumb structure of mix-breads to have some few big pores of an irregular shape, non-uniformly distributed pores and the rough crumb (Figure 4). Blue maize mix-bread had the highest chewiness and the lowest volume, which is in accordance with the above mentioned investigation [Eduardo et al., 2013]. On the contrary, the wheat bread had the softest crumb (696 g), the highest loaf volume $(232 \mathrm{~mL})$ and the chewiness of $461 \mathrm{~g}$ (Figures $3 \mathrm{a}, 3 \mathrm{~b}$ and $3 \mathrm{c}$ ). The texture profile analysis showed that the addition of blue and darkred popping maize flour to wheat flour significantly decreased springiness, cohesiveness and resilience of bread crumbs. Cohesiveness, resilience and springiness were decreased by 42 , 53 and $20 \%$ for blue maize mix-bread and by 30,40 and $16 \%$ for dark-red maize mix-bread compared with the properties of wheat bread, respectively (Figures $3 \mathrm{~d}$, $3 \mathrm{e}$ and $3 \mathrm{f}$ ).

The sensory evaluation showed differences between wheat bread and maize mix-breads (Figure 5). Although numer- ous phenolic compounds with important health benefits are characterized by bitterness and astringency [Lesschaeve \& Noble, 2005], the obtained results indicated that the wholegrain maize flour affected bread flavor providing a pleasant smell and taste and very intensive aroma. Mix-bread made by replacement of $30 \%$ of wheat flour with phenolicrichest dark-red maize flour had the best sensory quality in terms of the aforementioned properties. In contrast, results of Holtekjølen et al. [2008] indicated that mix-bread, made with barley flour contained the highest amount of phenolic compounds, had a lower acceptance among consumers than the other barley mix-breads and showed the highest score for bitterness, off-odor and off-flavor. The comparison of the results gained by the texture analysis (Figure 3) with the results obtained by sensory analysis (Figure 5) shows that the sensory analysis did not correlate with the instrumental parameters. Namely, blue maize mix-bread had the highest chewiness $(544.7 \mathrm{~g})$ and the lowest volume (187.7 mL) (Figure 3), while the sensory evaluation showed the best chewiness (4.6) (Figure 5). This shows that the sensory evaluation of bread

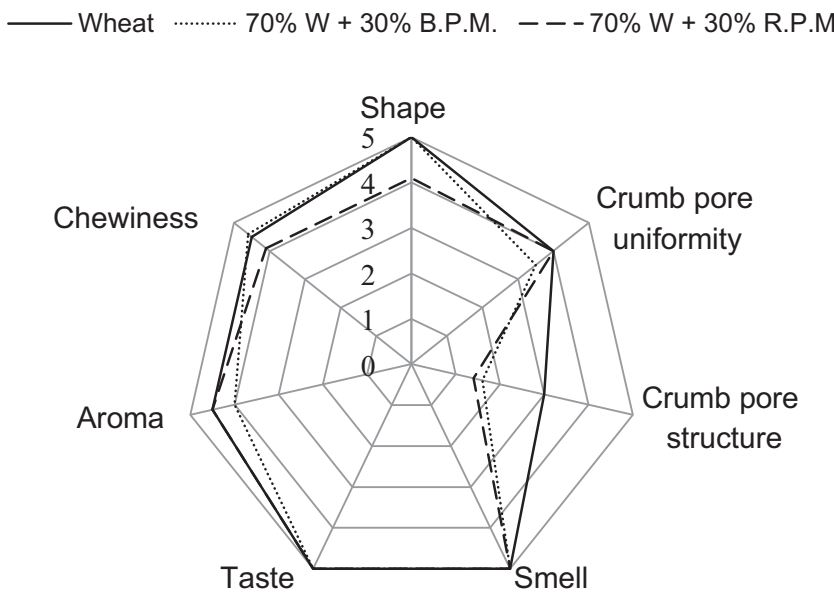

FIGURE 5. Spider web diagram of the sensory evaluation of wheat bread and maize mix-breads. W- wheat; B.P.M. - blue popping maize; R.P.M. dark-red popping maize. 
is a very sensitive task, often giving contradictory results, even with the highly experienced and trained sensory panel.

\section{CONCLUSION}

The replacement of $30 \%$ of wheat flour with wholegrain blue and dark-red popping maize flour, rich in phenolic compounds with biological activity, improved the functional profiles of end products increasing the content of total phenolics compounds, anthocyanins, and phenolic acids in maize mix-bread. Despite thermal degradation during baking, anthocyanins from maize flour could significantly contribute to the health benefits of bread, as well as its attractive color. Given to the synergistic and antagonistic interaction that arises from the coexistence of numerous antioxidant compounds in food, anthocyanin-rich maize flour had no effect on the increase in the bread antioxidant capacity. The Maillard reaction had a crucial influence on the increment of antioxidant capacity in wheat bread, as well as maize mix-breads.

The maize flour, 30\% which was added, had an unfavorable effect on all texture properties of breads, while their sensory quality was improved. The obtained results indicated that the wholegrain maize flour affected bread flavor providing a pleasant taste and very intensive aroma.

\section{RESEARCH FUNDING}

This study was supported by the Ministry of Education, Science and Technological Development of the Republic of Serbia (Grant no. TR 31069).

\section{CONFLICT OF INTERESTS}

Authors declare no conflict of interests.

\section{REFERENCES}

1. Abdel-Aal E.S.M., Hucl P., A rapid method for quantifying total anthocyanins in blue aleurone and purple pericarp wheats. Cereal Chem., 1999, 76, 350-354.

2. Bąkowska-Barczak A., Acylated anthocyanins as stable, natural food colorants - a review. Pol. J. Food Nutr. Sci., 2005, 14/55, 107-116.

3. Dangles O., Saito N., Brouillard R., Anthocyanin intramolecular copigment effect. Phytochemistry, 1993, 34, 119-124.

4. dePascual-Teresa S., Santos-Buelga C., Rivas-Gonzalo J.C., LCMS analysis of anthocyanins from purple corn cob. J. Sci. Food Agr., 2002, 82, 1003-1006.

5. Dey T.B., Chakraborty S., Jain K.K., Sharma A., Kuhad R.C., Antioxidant phenolics and their microbial production by submerged and solid state fermentation process: A review. Trends Food Sci. Tech., 2016, 53, 60-74.

6. Eberhardt M.V., Lee C.Y., Liu R.H., Antioxidant activity of fresh apples. Nature, 2000, 405, 903-904.

7. Eduardo M., Svanberg U., Oliveira J., Ahrné L., Effect of cassava flour characteristics on properties of cassava-wheat-maize composite bread types. Int. J. Food Sci., 2013, 2013, 1-10, art. id. 305407.
8. El-Megeid A.A.A., AbdAllah I.Z.A., Elsadek M.F., El-Moneim Y.F.A., The protective effect of the fortified bread with green tea against chronic renal failure induced by excessivedietary arginine in male albino rats. World J. Dairy Food Sci., 2009, 4, 107-117.

9. Filipčev B., Lević LJ., Bodroža-Solarov M., Mišljenović N., Koprivica G., Quality characteristics and antioxidant properties of breads supplemented with sugar beet molasses-based ingredients. Int. J. Food Prop., 2010, 13, 1035-1053.

10. Hofmann T., Characterization of the chemical structure of novel colored Maillard reaction products from furan-2-carboxaldehyde and amino acids. J. Agric. Food Chem., 1998, 46, 932-940.

11. Holtekjølen A.K., Bævre A.B., Rødbotten M., Berg H., Knutsen S.H., Antioxidant properties and sensory profiles of breads containing barley flour. Food Chem., 2008, 110, 414-421.

12. Hooper L., Cassidy A., A review of the health care potential of bioactive compounds. J. Sci. Food Agr., 2006, 86, 1805-1813.

13. Irmak S., Jonnala R.S., MacRitchie F., Effect of genetic variation on phenolic acid and policosanol contents of Pegaso wheat lines. J. Cereal Sci., 2008, 48, 20-26.

14. Kocadağlı T., Žilić S., Göncüoğlu Taş N., Vančetović J., Dodig D., Gökmen V., Formation of $\alpha$-dicarbonyl compounds in cookies made from wheat, hull-less barley and colored corn and its relation with phenolic compounds, free amino acids and sugars. Eur. Food Res. Technol., 2016, 242, 51-60.

15. Lesschaeve I., Noble A.C., Polyphenols: factors influencing their sensory properties and their effects on food and beverage preferences. Am. J. Clin. Nutr., 2005, 81, 330S-335S.

16. Menga V., Fares C., Troccoli A., Cattivelli L., Baiano A., Effect of genotype, location and baking on the phenolic content and some antioxidant properties of cereal species. Int. J. Food Sci. Technol., 2010, 45, 7-16.

17. Montilla E.C., Hillebrand S., Antezana A., Winterhalter P., Soluble and bound phenolic compounds in different Bolivian purple corn (Zea mays L.) cultivars. J. Agric. Food Chem., 2011, 59, 7068-7074.

18. Mora-Rochín S., Gaxiola-Cuevas N., Gutierrez-Uribe J.A., Milan-Carrillo J., Milan-Noris E.M., Reyes-Moreno C., Serna-Saldivar S.O., Cuevas-Rodríguez E.O., Effect of traditional nixtamalization on anthocyanin content and profile in Mexican blue maize (Zea mays L.) landraces. LWT - Food Sci. Technol., 2016, 68, 563-569.

19. Pedreschi R., Cisneros-Zevallos L., Phenolic profiles of Andean purple corn (Zea mays L.). Food Chem., 2007, 100, 956-963.

20. Scalbert A., Williamson G., Dietary intake and bioavailability of polyphenols. J. Nutr., 2000, 130, 2073S-2085S.

21. Serpen A., Gökmen V., Pellegrini N., Fogliano V., Direct measurement of the total antioxidant capacity of cereal products. J. Cereal Sci., 2008, 48, 816-820.

22. Siddiq M., Nasir M., Ravi R., Butt M. S., Dolan K.D., Harte J.B., Effect of defatted maire germ flour addition on the physical and sensory quality of wheat bread. LWT - Food Sci. Technol., 2009, 42, 464-470.

23. Singleton V.L., Orthofer R., Lamuela-Raventos R.M., Analysis of total phenols and other oxidation substrates and antioxidants by means of Folin-Ciocalteu reagent. Oxidants and Antioxidants, PTA Book Series: Methods Enzymol., 1999, 299, 152-178.

24. Stintzing F.C., Stintzing A.S., Carle R., Frei B., Wrolstad R.E., Color and antioxidant properties of cyanidin-based anthocyanin pigments. J. Agric. Food Chem., 2002, 50, 6172-6181. 
25. Świeca M., Gawlik-Dziki U., Dziki D., Baraniak B., Wheat bread enriched with green coffee - In vitro bioaccessibility and bioavailability of phenolics and antioxidant activity. Food Chem., 2017, 221, 1451-1457.

26. West E.M., Mauer J.L., Color and chemical stability of a variety of anthocyanins and ascorbic acid in solution and powder forms. J. Agric. Food Chem., 2013, 61, 4169-4179.

27. $\mathrm{Xu} \mathrm{J.,} \mathrm{Identification} \mathrm{and} \mathrm{stability} \mathrm{of} \mathrm{acylated} \mathrm{anthocyanins}$ in purplefleshed sweetpotato. 2013, p40 [http://krex.k-state.edu/ dspace/handle/2097/16910].

28. Yousif A., Nhepera D., Johnson S., Influence of sorghum flour addition on flat bread in vitro starch digestibility, antioxidant capacity and consumer acceptability. Food Chem., 2012, 134, 880-887
29. Žilić S., Serpen A., Akillioglu G., Gökmen V., Vančetović J., Phenolic compounds, carotenoids, anthocyanins, and antioxidant capacity of colored maize (Zea mays L.) kernels. J. Agric. Food Chem., 2012, 60, 1224-1231.

30. Žilić S., Kocadagli T., Vančetović J., Gokmen V., Effects of baking conditions and dough formulations on phenolic compound stability, antioxidant capacity and color of cookies made from anthocyanin-rich corn flour. LWT - Food Sci. Technol., 2016, 65, 597-603.

Submitted: 15 August 2017. Revised: 22 January 2018. Accepted: 7 March 2018. Published on-line: 28 March 2018. 